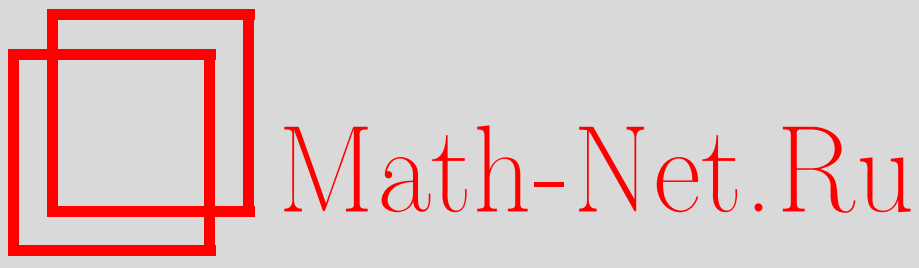

П. Е. Жидков, О базисности Рисса системы собственных функций нелинейной задачи типа Штурма-Лиувилля, Матем. сб., 2000, том 191, номер 3, 43-52

DOI: https://doi.org/10.4213/sm461

Использование Общероссийского математического портала Math-Net.Ru подразумевает, что вы прочитали и согласны с пользовательским соглашением http://www . mathnet.ru/rus/agreement

Параметры загрузки:

IP : 54.197 .217 .227

26 апреля 2023 г., 10:33:18 


\title{
П.Е. Жидков
}

\section{О базисности Рисса системы собственных функций нелинейной задачи типа Штурма-Лиувилля}

\begin{abstract}
Для нелинейной задачи на собственные значения, подобной линейной задаче Штурма-Лиувилля, исследованы свойства спектра и собственных функций и доказано, что система собственных функций является базисом Рисса в пространстве $L_{2}$.

Библиографйия: 10 названий.
\end{abstract}

\section{§1. Введение. Определения. Основной результат}

В работе рассматривается следующая нелинейная задача на собственные значения:

$$
\begin{gathered}
-y^{\prime \prime}+f\left(y^{2}\right) y=\lambda y, \quad \lambda \in(-\infty, \infty), \quad y=y(x), \quad x \in(0, A), \\
y(0)=p, \quad y^{\prime}(0)=y(A)=0 .
\end{gathered}
$$

Здесь все величины вещественны, $A$ и $p$ - фиксированные положительные параметры, $f\left(y^{2}\right) y$ - непрерывно дифференцируемая функция аргумента $y \in(-\infty, \infty)$, удовлетворяющая некоторьм дополнительным условиям, а $\lambda$-спектральный параметр. Если пара $(\lambda, y(x))$, где $y(x) \in C^{2}(0, A) \cap C^{1}[0, A) \cap C[0, A]$, удовлетворяет задаче (1)-(2), то назовем $\lambda$ собственным значением, а $y(x)$ - соответствующей собственной функиией задачи (1)-(2). Множество всех собственных значений назовем спектром задачи (1)-(2).

Имеется значительное количество статей, в которых устанавливается наличие счетных наборов собственных функций нелинейных задач типа (1)-(2) и значительно более сложных (см., например, [1; часть 2, гл. III, пा. 10.3, 11.4, 11.5] и библиографию там же). Целью настоящей работы является доказательство базисности в $L_{2}$ счетной системы собственных функций задачи (1)-(2).

Нелинейная задача на собственные значения, подобная задаче (1)-(2), рассматривалась автором в работе [2]. К сожалению, в формулировке и доказательстве теоремы 1 из работы [2] имеются ошибки, которые, однако, можно исправить. Автор надеется в недалеком будущем опубликовать эти исправления, а также некоторые дополнения к указанной работе. Кроме того, в [3] дано независимое доказательство результатов работы [2] для рассматриваемой в последней нелинейной задачи, основанное на теореме Бари (см. далее).

Введем некоторые обозначения, используемые в дальнейшем. Пусть $L_{2}(a, b)$, где $a<b,-$ пространство Лебега, состоящее из вещественных функций, квадратично интегрируемых по отрезку $[a, b]$, со скалярным произведением

$$
(g, h)_{L_{2}(a, b)}=\int_{a}^{b} g(x) h(x) d x
$$

(C) П.Е. Жидков 2000 
и нормой $\|g\|_{L_{2}(a, b)}=(g, g)_{L_{2}(a, b)}^{1 / 2}$. Хорошо известно, что $L_{2}(a, b)$ - гильбертово пространство. Положим $L_{2}=L_{2}(0, A),\|\cdot\|=\|\cdot\|_{L_{2}(0, A)}$ и $(\cdot, \cdot)=(\cdot, \cdot)_{L_{2}(0, A)}$.

Следующее определение общеизвестно.

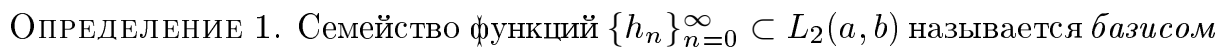
пространства $L_{2}(a, b)$, если для произвольной функции $h \in L_{2}(a, b)$ существует единственный набор вещественных коэффициентов $\left\{a_{n}\right\}_{n=0}^{\infty}$ такой, что

$$
\lim _{N \rightarrow \infty}\left\|\sum_{n=0}^{N} a_{n} h_{n}-h\right\|_{L_{2}(a, b)}=0
$$

последнее равенство будем записывать так: $\sum_{n=0}^{\infty} a_{n} h_{n}=h$, оговаривая, если необходимо, в смысле какого пространства оно понимается.

В соответствии с работами [4], [5] (см. также [6]-[8]) введем еще следующие определения.

ОПРЕДЕЛЕНИЕ 2. Базис $\left\{h_{n}\right\}_{n=0}^{\infty} \subset L_{2}(a, b)$ называется базисом Puсca, если для произвольной функции $h=\sum_{n=0}^{\infty} a_{n} h_{n} \in L_{2}(a, b)$, где $a_{n} \in(-\infty, \infty)$, $n=0,1,2, \ldots$, выполнено условие $\sum_{n=0}^{\infty} a_{n}^{2}<\infty$ и, наоборот, для любых вешественных чисел $a_{n}, n=0,1,2, \ldots$, таких, что $\sum_{n=0}^{\infty} a_{n}^{2}<\infty$, ряд $\sum_{n=0}^{\infty} a_{n} h_{n}$ сходится в пространстве $L_{2}(a, b)$.

ОПРЕДЕЛЕНИЕ 3. Система функций $\left\{h_{n}\right\}_{n=0}^{\infty} \subset L_{2}(a, b)$ назьвается минимальной, если для любого номера $k \geqslant 0$ существует $\delta>0$ такое, что

$$
\left\|\sum_{\substack{n=0 \\ n \neq k}}^{N} a_{n} h_{n}-h_{k}\right\|_{L_{2}(a, b)} \geqslant \delta
$$

для любого номера $N>0$ и любых вещественных коэффициентов $a_{n}$.

Следующий результат Н. К. Бари, используемый при доказательстве основного результата настояшей работы, анонсирован в [4] и доказан в [5] (см. также [6]-[8]).

ТЕОРемА БАРИ. Пусть $\left\{e_{n}\right\}_{n=0}^{\infty}$ - базис Pисса в пространстве $L_{2}(a, b)$, u пусть система функиий $\left\{h_{n}\right\}_{n=0}^{\infty} \subset L_{2}(a, b)$ удовлетворяет следующим условиям:

А. система функиий $\left\{h_{n}\right\}_{n=0}^{\infty}$ минимальна в $L_{2}(a, b)$;

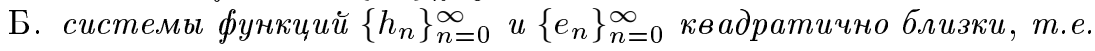

$$
\sum_{n=0}^{\infty}\left\|h_{n}-e_{n}\right\|_{L_{2}(a, b)}^{2}<\infty .
$$

Тогда система $\left\{h_{n}\right\}_{n=0}^{\infty}$ является базисом Рисса в пространстве $L_{2}(a, b)$.

В действительности в монографиии [7] доказано несколько более сильное утверждение, однако доказательство из работы [5] остается в силе и в этом случае. Базисы, квадратично близкие к ортонормированным, называют также базисами Бари (см. [7]). По поводу свойств базисов Рисса и Бари см. [4]-[8].

Основной результат работы составляет 
ТЕОРЕма 1. Пусть $f\left(y^{2}\right) y$ - непрерывно дифференцируемая функиия аргумента $у \in(-\infty, \infty)$, и пусть $f(s)$ - монотонно неубывающая функция аргумента $s \in[0,+\infty)$. Тогда

(а) для любого иелого $n \geqslant 0$ существует единственная пара $\left(\lambda_{n}, y_{n}(x)\right)$, состоящая из собственного значения $\lambda_{n}$ и соответствующей собственной функиии $y_{n}(x)$ задачи (1)-(2), такая, что функиия $y_{n}(x)$ имеет ровно $n$ корней в интервале $(0, A)$;

(б) для любого номера $n$ корнями функиии $y_{n}(x)$ в интервале $(0, A)$ являются точки $\frac{(2 k+1) A}{2 n+1}$, где $k=\overline{0, n-1}$;

(в) $f\left(p^{2}\right)<\lambda_{0}<\lambda_{1}<\cdots<\lambda_{n}<\cdots u \lim _{n \rightarrow \infty} \lambda_{n}=+\infty$;

(г) система собственных функиий $\left\{y_{n}\right\}_{n=0}^{\infty}$ является базисом Рисса в пространстве $L_{2}$, а система функиий $\left\{\sqrt{2 / A} p^{-1} y_{n}\right\}_{n=0}^{\infty}-$ базисом Бари в том же пространстве.

Очевидно, результат, аналогичный теореме 1 , имеет место и для задачи, в которую входят уравнение (1) и граничные условия

$$
y(0)=y^{\prime}(A)=0, \quad y(A)=p,
$$

поскольку задача (1)-(2) сводится к последней заменой $x \rightarrow A-x$. В настоящей работе автор не ставил себе целью рассмотреть как можно более широкий класс задач, к которым применим используемый здесь подход, основанный на проверке условий теоремы Бари, а хотел лишш проиллюстрировать возможность использования этого подхода на примере конкретной задачи (1)-(2).

Предположения теоремы 1 выполнены, например, для задачи (1)-(2) с $f(s)=$ $|s|^{q}$, где $q \geqslant 0$ - постоянная. В частности, при $q=1$ уравнение (1) становится вариантом уравнения Дуффинга. Уравнение (1) в общем случае интегрируемо в квадратурах, однако автор не знает нетривиальных примеров функции $f$, при которых собственные функции задачи (1)-(2) выражались бы в элементарных функциях.

Что касается приложений полученных результатов, то, по мнению автора, можно надеяться, что базисы, состоящие из решений нелинейных краевых задач, в дальнейшем могут быть использованы, например, в методе Галёркина. По сведениям автора настоящая работа является одной из первых статей, в которых доказана базисность подобной системы. Отметим монографию [8], в которой представлены некоторые интересные результаты в этой области.

\section{§ 2. Доказательство теоремы 1}

Рассмотрим задачу Коши

$$
\begin{gathered}
-u^{\prime \prime}+f\left(u^{2}\right) u=\lambda u, \quad u=u(x), \quad x \in(-\infty, \infty), \\
u(0)=p, \quad u^{\prime}(0)=0 .
\end{gathered}
$$

В силу условий теоремы 1 для нее справедливы обычные локальные теоремы сушествования, единственности и непрерывной зависимости решения $u(x)$ от параметра $\lambda$.

Рассмотрим произвольное $\lambda<f\left(p^{2}\right)$. Тогда в силу уравнения $(3) u^{\prime \prime}(0)>0$, поэтому $u(x)>p$ и $u^{\prime}(x)>0$ для любого достаточно малого $x>0$. Но тогда, поскольку $f(s)$ - неубывающая функция аргумента $s \in[0,+\infty)$, в силу уравнения (3) 
$u(x)>p, u^{\prime}(x)>0$ и $u^{\prime \prime}(x)>0$ для всех $x>0$, для которых решение $u(x)$ определено. Действительно, предположим противное и возьмем точную нижнюю грань $x_{0}$ точек $x>0$, при которых хотя бы одно из этих неравенств нарушается. В силу сказанного $x_{0}>0$ и $u(x)>p, u^{\prime}(x)>0$ и $u^{\prime \prime}(x)>0$ при всех $x \in\left(0, x_{0}\right)$. Отсюда по формуле Ньютона-Лейбница получаем $u\left(x_{0}\right)>p$ и $u^{\prime}\left(x_{0}\right)>0$, поэтому в силу уравнения $(3) u^{\prime \prime}\left(x_{0}\right)>0$. Следовательно, ввиду непрерывности эти неравенства вьполняются в некоторой правой полуокрестности точки $x_{0}$, т.е. получаем противоречие с определением этой точки. При $\lambda=f\left(p^{2}\right)$ в силу теоремы единственности $u(x) \equiv p$. Таким образом, точки $\lambda \leqslant f\left(p^{2}\right)$ не принадлежат спектру задачи $(1)-(2)$.

Для решений задачи (3)-(4), очевидно, справедливо тождество

$$
\left\{\left[u^{\prime}(x)\right]^{2}+\lambda u^{2}(x)-F\left(u^{2}(x)\right)\right\}^{\prime}=0,
$$

где $F(s)=\int_{0}^{s} f(r) d r$, из которого следует, что

$$
\lambda p^{2}-F\left(p^{2}\right)=\left[u^{\prime}(x)\right]^{2}+\lambda u^{2}(x)-F\left(u^{2}(x)\right)
$$

для всех $x$, для которых решение $u(x)$ определено.

Пусть $\lambda>f\left(p^{2}\right)$. Тогда в силу уравнения (3) и тождества (5) $u(x) u^{\prime \prime}(x)<0$ и $u^{\prime}(x)=0$ для любого $x$ такого, что $|u(x)|=p$. Следовательно, для $\lambda>f\left(p^{2}\right)$ имеет место априорная оценка решения $u(x)$ задачи $(3)-(4)$ :

$$
|u(x)| \leqslant p
$$

для всех $x$, для которых это решение определено. Действительно, предположим противное и обозначим через $x_{0} \geqslant 0$ точную нижнюю грань значений $x>0$, при которых это неравенство нарушается. Тогда $\left|u\left(x_{0}\right)\right|=p$, поэтому $u\left(x_{0}\right) u^{\prime \prime}\left(x_{0}\right)<0$ и $u^{\prime}\left(x_{0}\right)=0$, а отсюда по формуле Тейлора легко следует, что $|u(x)|<p$ в некоторой правой полуокрестности точки $x_{0}$; получили противоречие. Из (6), тождества (5) и уравнения (3) вытекает, что для любого $\lambda>f\left(p^{2}\right)$ сушествует $C_{1}>0$ такое, что

$$
\left|u^{\prime}(x)\right| \leqslant C_{1} \quad \text { и }\left|u^{\prime \prime}(x)\right| \leqslant C_{1}
$$

для всех $x$, для которых решение $u(x)$ определено.

Из оценок (6) и (7) сразу следует, что при $\lambda>f\left(p^{2}\right)$ решение $u(x)$ задачи $(3)-(4)$ может быть продолжено для всех $x \in(-\infty, \infty)$. Действительно, предположим, что сушествуют $\lambda>f\left(p^{2}\right)$ и $d>0$ такие, что соответствуюшее решение $u(x)$ задачи (3)-(4) может быть продолжено на полуинтервал $[0, d)$ и не может быть продолжено ни на одну правую полуокрестность точки $d$. Положим

$$
c=\lim _{x \rightarrow d-0} u(x)=p+\int_{0}^{d} u^{\prime}(x) d x, \quad c^{\prime}=\lim _{x \rightarrow d-0} u^{\prime}(x)=\int_{0}^{d} u^{\prime \prime}(x) d x
$$

и рассмотрим задачу Коши для уравнения (3) с начальными условиями $u(d)=c$, $u^{\prime}(d)=c^{\prime}$. Она локально однозначно разрешима, откуда немедленно вытекает, что решение $u(x)$ задачи (3)-(4) может быть продолжено на некоторую правую полуокрестность точки $x=d$, т.е. получаем противоречие. Следовательно, решение $u(x)$ может быть продолжено на полупрямую $[0,+\infty)$. Кроме того, очевидно, 
что функция, определенная равенством $u(-x)=u(x)$ при $x \geqslant 0$, удовлетворяет задаче (3)-(4) при всех $x \in(-\infty, \infty)$. Тем самым доказано, что при $\lambda>f\left(p^{2}\right)$ решение задачи (3)-(4) может быть продолжено на всю прямую $x \in(-\infty, \infty)$. В дальнейшем будем считать, что при $\lambda>f\left(p^{2}\right)$ соответствуюшее решение $u(x)$ задачи (3)-(4) продолжено на всю прямую $x \in(-\infty, \infty)$.

Возьмем произвольное $\lambda>f\left(p^{2}\right)$. Тогда для соответствуюшего решения $u(x)$ задачи (3)-(4) в силу уравнения (3) выполнено $u^{\prime \prime}(0)<0$, поэтому найдется $d>0$ такое, что $u(x)>0$ и $u^{\prime}(x)<0$ при $x \in(0, d]$. Далее, поскольку $U(s)=\lambda s^{2}-F\left(s^{2}\right)$ - возрастающая функция аргумента $s \in[0, p]\left(\right.$ так как $\frac{d}{d s} U(s)=2 \lambda s-2 s f\left(s^{2}\right)>0$ при $s \in(0, p])$, из тождества (5) следует, что для любого $\varepsilon \in(0, p)$ сушествует $a=a(\lambda)>0$ такое, что $\left|u^{\prime}(x)\right| \geqslant a$, если $u(x) \in(-p+\varepsilon, p-\varepsilon)$. (Это так, поскольку в силу предыдущего для любого $\varepsilon>0$ найдется положительное $a$ такое, что $\lambda p^{2}-$ $F\left(p^{2}\right)-\left[\lambda u^{2}-F\left(u^{2}\right)\right] \geqslant a^{2}$ для всех $u \in(-p+\varepsilon, p-\varepsilon)$.) Поэтому решение $u(x)$ имеет положительный корень. Для каждого $\lambda>f\left(p^{2}\right)$ через $x_{0}(\lambda)$ обозначим минимальный положительный корень решения $u(x)$ задачи $(3)-(4)$. Отметим, что из приведенных выше рассуждений следует, что $u^{\prime}(x)<0$ при $x \in\left(0, x_{0}(\lambda)\right]$.

ЛЕмма 1. Функиия $x_{0}(\lambda)$ является строго убивающей непрерывной функиией аргумента $\lambda \in\left(f\left(p^{2}\right),+\infty\right)$. При этом $\lim _{\lambda \rightarrow f\left(p^{2}\right)+0} x_{0}(\lambda)=+\infty, a$ $\lim _{\lambda \rightarrow+\infty} x_{0}(\lambda)=0$.

ДокаЗАтЕльство. Свойство $\lim _{\lambda \rightarrow f\left(p^{2}\right)+0} x_{0}(\lambda)=+\infty$ следует из того, что при $\lambda=f\left(p^{2}\right)$ решением задачи (3)-(4) является функция $u(x) \equiv p$, и из теоремы о непрерывной зависимости этого решения от параметра $\lambda$. Из оценки (6) и из теоремы сравнения (см. [9]) следует свойство $\lim _{\lambda \rightarrow+\infty} x_{0}(\lambda)=0:$ надо сравнить задачу (3)-(4) с задачей

$$
-w^{\prime \prime}=\mu w, \quad w=w(x), \quad w(0)=p, \quad w^{\prime}(0)=0
$$

взятой при достаточно больших значениях параметра $\mu>0$.

Докажем непрерывность функции $x_{0}(\lambda)$. Фиксируем произвольное $\bar{\lambda}>f\left(p^{2}\right)$ и обозначим через $u(\lambda, x)$ решение задачи (3)-(4). Поскольку $\left.u_{x}^{\prime}(\bar{\lambda}, x)\right|_{x=x_{0}(\bar{\lambda})} \neq 0$ (иначе в точке $x_{0}(\bar{\lambda})$ нарушалась бы теорема единственности, так как функция $u(x) \equiv 0$ является решением задачи Коши для уравнения (3) с начальными условиями $\left.u\left(x_{0}(\bar{\lambda})\right)=\left.u^{\prime}(x)\right|_{x=x_{0}(\bar{\lambda})}=0\right)$, по теореме о неявной функции существуют $\varepsilon \in\left(0, x_{0}(\bar{\lambda})\right)$ и $\delta \in\left(0, \bar{\lambda}-f\left(p^{2}\right)\right)$ такие, что для каждого $\lambda$, удовлетворяюшего условию $|\lambda-\bar{\lambda}|<\delta$, сушествует единственное $x_{1}=x_{1}(\lambda)$ такое, что $x_{1}(\lambda) \in\left(x_{0}(\bar{\lambda})-\varepsilon, x_{0}(\bar{\lambda})+\varepsilon\right)$ и $u\left(\lambda, x_{1}(\lambda)\right)=0$, причем функция $x_{1}(\lambda)$ непрерьвна при $\lambda \in(\bar{\lambda}-\delta, \bar{\lambda}+\delta)$. Далее, поскольку $\min _{x \in\left[0, x_{0}(\bar{\lambda})-\varepsilon\right]} u(\bar{\lambda}, x)>0$, для всех $\lambda$, достаточно близких к $\bar{\lambda}, u(\lambda, x)$ как функция аргумента $x$ не имеет корней на отрезке $\left[0, x_{0}(\bar{\lambda})-\varepsilon\right]$, т.е. $x_{1}(\lambda)=x_{0}(\lambda)$ для всех значений параметра $\lambda$, достаточно близких к $\bar{\lambda}$. Тем самым непрерывность функции $x_{0}(\lambda)$ доказана.

Докажем монотонность функции $x_{0}(\lambda)$. Возьмем произвольные $\lambda_{1}, \lambda_{2}: f\left(p^{2}\right)<$ $\lambda_{1}<\lambda_{2}$. Обозначим через $u_{1}(x)$ и $u_{2}(x)$ соответствуюшие решения задачи $(3)-(4)$. Пусть $d=\min \left\{x_{0}\left(\lambda_{1}\right) ; x_{0}\left(\lambda_{2}\right)\right\}$. Тогда в силу указанного выше свойства $u_{i}^{\prime}(x)<0$ 
при $x \in\left(0, x_{0}\left(\lambda_{i}\right)\right]$ и тождества $(5)$ для любых $x_{1}, x_{2} \in(0, d]$ таких, что $u_{1}\left(x_{1}\right)=$ $u_{2}\left(x_{2}\right)$, имеем

$$
\begin{aligned}
u_{2}^{\prime}\left(x_{2}\right) & =-\left\{\lambda_{2} p^{2}-F\left(p^{2}\right)-\lambda_{2} u_{2}^{2}\left(x_{2}\right)+F\left(u_{2}^{2}\left(x_{2}\right)\right)\right\}^{1 / 2} \\
& <-\left\{\lambda_{1} p^{2}-F\left(p^{2}\right)-\lambda_{1} u_{1}^{2}\left(x_{1}\right)+F\left(u_{1}^{2}\left(x_{1}\right)\right)\right\}^{1 / 2}=u_{1}^{\prime}\left(x_{1}\right)<0 .
\end{aligned}
$$

Кроме того, в силу уравнения $(3) u_{2}^{\prime \prime}(0)<u_{1}^{\prime \prime}(0)<0$, поэтому $u_{2}(x)<u_{1}(x)<p$ в некоторой правой полуокрестности точки $x=0$. Следовательно, $x_{0}\left(\lambda_{1}\right)>x_{0}\left(\lambda_{2}\right)$, в противном случае графики функций $u_{1}(x)$ и $u_{2}(x)$ должны были бы пересекаться в полуинтервале $(0, d]$, а в минимальной точке $\bar{x} \in(0, d]$ такого пересечения выполнялось бы $u_{1}^{\prime}(\bar{x}) \leqslant u_{2}^{\prime}(\bar{x})$, что противоречит предыдушему. Тем самым лемма 1 доказана.

ЛЕмма 2. Пусть $\lambda>f\left(p^{2}\right)$ произвольно и $и(x)$ - соответствующее решение задачи (3)-(4). Тогда

1) $u(-x)=u(x)$ для любого $x \in(-\infty, \infty)$;

2) $u\left(2 x_{0}(\lambda)+x\right)=-u(x)$ для любого $x \in(-\infty, \infty)$.

ДокАЗАТЕЛьство следует из локальных теорем существования и единственности для задачи (3)-(4), автономности уравнения (3) и его инвариантности относительно замен переменных $u(x) \rightarrow-u(x)$ и $x \rightarrow c-x$, где $c-$ произвольная вешественная постоянная. Действительно, свойство 1) следует из того, что, как нетрудно проверить, если $u(x)$ - решение задачи (3)-(4), то $u(-x)$ - также решение этой задачи, и из теоремы единственности. Совершенно аналогично доказывается, что решение $u(x)$ четно относительно любой точки $x$ такой, что $u^{\prime}(x)=0$, и нечетно относительно любого своего корня. Докажем, например, последнее утверждение. Пусть $x_{0}-$ корень функции $u(x)$. Для $x \leqslant x_{0}$ положим $v(x)=-u\left(x_{0}+\left(x_{0}-x\right)\right)$. Тогда легко можно проверить, что функция $v(x)$ является решением уравнения $(3)$ на полупрямой $x \leqslant x_{0}$, причем $v\left(x_{0}\right)=0$ и $v^{\prime}\left(x_{0}-0\right)=u^{\prime}\left(x_{0}\right)$. Поэтому по теореме единственности решение $u(x)$ совпадает с функцией $v(x)$ при всех $x \leqslant x_{0}$, и нечетность решения $u(x)$ задачи $(3)-(4)$ относительно любого своего корня доказана.

Итак, считаем доказанным, что решение $u(x)$ четно относительно точки $x=0$ и относительно любой точки $x$, в которой $u^{\prime}(x)=0$, и что оно нечетно относительно любого своего корня. Поэтому решение $u(x)$ можно построить следуюшим образом. С отрезка $\left[0, x_{0}(\lambda)\right]$ на отрезок $\left[x_{0}(\lambda), 2 x_{0}(\lambda)\right]$ продолжаем функцию $u(x)$ нечетно относительно точки $x_{0}(\lambda)$. Далее, поскольку для построенной функции $u^{\prime}\left(2 x_{0}(\lambda)-0\right)=u^{\prime}(0)=0$, продолжаем ее четно относительно точки $2 x_{0}(\lambda)$ на отрезок $\left[2 x_{0}(\lambda), 4 x_{0}(\lambda)\right]$. Продолжая эти рассуждения, легко убеждаемся, что далее нужно продолжить построенную функцию на всю вешественную прямую периодически с периодом $4 x_{0}(\lambda)$; построенная таким образом функция и будет решением задачи (3)-(4). Тем самым утверждение 2 ), а вместе с ним и лемма 2 , доказано.

Фиксируем произвольное целое $n \geqslant 0$. В силу леммы 1 существует ровно одно значение параметра $\lambda \in(-\infty, \infty)$, причем $\lambda>f\left(p^{2}\right)$ ввиду рассуждений из начала этого параграф̆а, такое, что $(2 n+1) x_{0}(\lambda)=A$. Ввиду лемм 1 и 2 ясно также, что при этом значении параметра $\lambda$ точки $(2 k+1) x_{0}(\lambda)$, где $k=\overline{0, n}$, и только они являются корнями соответствуюшего решения $u(x)$ задачи $(3)-(4)$ в полуинтервале $(0, A]$ и что при любом другом значении параметра $\lambda$ либо число корней 
соответствуюшего решения $u(x)$ задачи $(3)-(4)$ в интервале $(0, A)$ не равно $n$, либо $u(A) \neq 0$. Тем самым доказаны утверждения (а) и (б) теоремы 1. Свойства $\lambda_{0}<\cdots<\lambda_{n}<\cdots$ и $\lim _{n \rightarrow \infty} \lambda_{n}=+\infty$ следуют из этих же рассуждений и леммы 1. Таким образом, утверждение (в) теоремы 1 также доказано.

Приступим к доказательству утверждения (г). Рассмотрим линейную задачу на собственные значения

$$
-e^{\prime \prime}=z e, \quad x \in(0, A), e=e(x), \quad e^{\prime}(0)=e(A)=0
$$

(здесь $z$ - спектральный параметр). Тогда $z_{n}=\left(\frac{\pi(2 n+1)}{2 A}\right)^{2}-$ ее собственные значения, а $e_{n}(x)=\sqrt{\frac{2}{A}} \cos \frac{\pi(2 n+1) x}{2 A}-$ соответствующие нормированные в пространстве $L_{2}$ собственные функции (здесь $n=0,1,2, \ldots$ ), причем система собственных функций $\left\{e_{n}\right\}_{n=0}^{\infty}$ является ортонормированным базисом в пространстве $L_{2}$. В частности, очевидно, эта система есть базис Рисса в указанном пространстве. Далее, для каждого целого $n \geqslant 0$ положим $g_{n}(x)=p \sqrt{A / 2} e_{n}(x)$. Для доказательства утверждения (г) теоремы 1 достаточно проверить условия А и Б теоремы Бари для системы функций $\left\{w_{n}\right\}_{n=0}^{\infty}$, где $w_{n}(x)=p^{-1} \sqrt{2 / A} y_{n}(x)$, взяв в качестве базиса Рисса систему $\left\{e_{n}\right\}_{n=0}^{\infty}$. Отметим еще, что условие Б теоремы Бари равносильно тому, что $\sum_{n=0}^{\infty}\left\|g_{n}-y_{n}\right\|^{2}<\infty$.

Для каждого целого $n \geqslant 0$ разложим функцию $w_{n}\left(\frac{x}{2 n+1}\right)$ в ряд Фурье в пространстве $L_{2}$ :

$$
w_{n}\left(\frac{x}{2 n+1}\right)=\sum_{m=0}^{\infty} a_{m}^{n} e_{m}(x)
$$

где $a_{m}^{n}$ - вещественные коэффициенты. Поскольку $w_{n}\left(\frac{x}{2 n+1}\right)>0$ и $e_{0}(x)>0$ при $x \in[0, A)$, имеем $a_{0}^{n}=\left(w_{n}\left(\frac{\cdot}{2 n+1}\right), e_{0}(\cdot)\right)>0$. Далее, из разложения $(8)$ получаем:

$$
w_{n}(x)=\sum_{m=0}^{\infty} b_{n, m} e_{m}(x)
$$

причем $b_{n, l_{0}(m)}=a_{m}^{n}, m=0,1,2, \ldots$, и $b_{n, l}=0$, если $l \neq l_{0}(m)$ для всех целых $m \geqslant 0$, где $l_{0}(m)=((2 m+1)(2 n+1)-1) / 2$ (в частности, $b_{n, 0}=\cdots=b_{n, n-1}=0$ и $\left.b_{n, n}=a_{0}^{n}>0\right)$, и ряд Фурье из правой части (9) сходится в пространстве $L_{2}\left(0, \frac{A}{2 n+1}\right)$.

Лемма 3. Ряд Фурье из правой части (9) сходится к функиии $w_{n}$ в пространстве $L_{2}=L_{2}(0, A)$.

ДоказАТЕЛьство. Как отмечалось выше, равенство (9) имеет место в смысле пространства $L_{2}\left(0, \frac{A}{2 n+1}\right)$. В силу четности функций $w_{n}(x)$ и $e_{l_{0}(m)}(x)$ для произвольного целого $m \geqslant 0$ оно также выполнено в пространстве $L_{2}\left(-\frac{A}{2 n+1}, 0\right)$. 
Кроме того, в правой части (9) могут быть отличными от нуля лишь коэффициенты $b_{n, m}$ с $m=l_{0}(l)$ для некоторого целого $l \geqslant 0$. Для произвольных $x \in\left(-\frac{A}{2 n+1}, \frac{A}{2 n+1}\right)$, целого $l \geqslant 0$ и $k=1, \ldots, n$ в силу леммы 2 имеем $w_{n}\left(x+\frac{2 k A}{2 n+1}\right)=(-1)^{k} w_{n}(x)$ и

$$
\begin{gathered}
e_{l_{0}(l)}\left(x+\frac{2 k A}{2 n+1}\right)=\sqrt{\frac{2}{A}} \cos \left[\frac{\pi(2 l+1)(2 n+1)(x+2 k A /(2 n+1))}{2 A}\right] \\
=(-1)^{k} \sqrt{\frac{2}{A}} \cos \frac{\pi(2 l+1)(2 n+1) x}{2 A}=(-1)^{k} e_{l_{0}(l)}(x) .
\end{gathered}
$$

Следовательно, в каждом из пространств $L_{2}\left(\frac{(2 k+1) A}{2 n+1}, \frac{(2 k+3) A}{2 n+1}\right), k=\overline{0, n-1}$, равенство (9) также выполнено (формальное доказательство этого утверждения очевидно). Тем самым лемма 3 доказана.

Докажем минимальность системы $\left\{w_{n}\right\}_{n=0}^{\infty}$ в пространстве $L_{2}$. Предположим, что эта система не является минимальной. Тогда существуют номер $N \geqslant 0$, последовательности номеров $N_{k}>0$, вешественных коэффициентов $\left\{d_{n}^{k}\right\}_{n=0}^{N_{k}}$ и функций $\alpha_{k}(x)$, где $k=1,2,3, \ldots$, такие, что

$$
w_{N}+\sum_{\substack{n=0 \\ n \neq N}}^{N_{k}} d_{n}^{k} w_{n}=\alpha_{k}, \quad \text { где }\left\|\alpha_{k}\right\| \rightarrow 0 \text { при } k \rightarrow \infty .
$$

В дополнение можно считать, что $\left\{N_{k}\right\}_{k=1}^{\infty}$ - монотонно возрастающая последовательность номеров и $N_{1}>N$, вводя дополнительно нулевые коэффициенты $d_{n}^{k}$, если необходимо. Обозначим через $P_{m}$ ортогональный проектор в пространстве $L_{2}$ на подпространство, натянутое на векторы $e_{0}, \ldots, e_{m}$, а через $P_{m}^{\perp}-$ ортогональный проектор в том же пространстве на замыкание линейной оболочки векторов $\left\{e_{k}\right\}_{k=m+1}^{\infty}$. Предполагая, что $N>0$, положим $\alpha_{k}^{N-1}=P_{N-1} \alpha_{k}$. Тогда, поскольку $\left\|P_{m} w\right\| \leqslant\|w\|$ для любого $w \in L_{2}$ и любого номера $m$, имеем $\left\|\alpha_{k}^{N-1}\right\| \rightarrow 0$ при $k \rightarrow \infty$. Далее, ввиду разложений (9) и леммы $3 \alpha_{k}^{N-1}=\sum_{n=0}^{N-1} d_{n}^{k} P_{N-1} w_{n}$, где в силу того что, как указано выше, матрица $\left(b_{n, m}\right)_{n, m=0}^{N-1}-$ верхнетреугольная и $b_{n, n}>0$ для любого номера $n$, векторы $\left\{P_{N-1} w_{n}\right\}_{n=0}^{N-1}-$ ненулевые и линейно независимые. Отсюда по известной теореме об эквивалентности любых двух норм в конечномерном линейном пространстве (см. [10]) сразу следует, что $d_{n}^{k} \rightarrow 0$ при $k \rightarrow \infty$ для всех $n=\overline{0, N-1}$. Поэтому получаем

$$
w_{N}+\sum_{n=N+1}^{N_{k}} d_{n}^{k} w_{n}=\beta_{k}, \quad \text { где }\left\|\beta_{k}\right\| \rightarrow 0 \text { при } k \rightarrow \infty .
$$

Если $N=0$, то последнее соотношение также очевидно выполняется. Но в силу разложений (9) и леммы $3 \sum_{n=N+1}^{N_{k}} d_{n}^{k} w_{n} \in P_{N}^{\perp} L_{2}$, поэтому

$$
\left\|w_{N}+\sum_{n=N+1}^{N_{k}} d_{n}^{k} w_{n}\right\| \geqslant b_{N, N}
$$


следовательно, поскольку $b_{N, N}>0$ не зависит от номера $k$, получаем противоречие. Тем самьм доказано, что система $\left\{w_{n}\right\}_{n=0,1,2, \ldots}-$ минимальная в пространстве $L_{2}$.

Проверим условие Б теоремы Бари.

Лемма 4. Существует постоянная $C_{2}>0$ такая, что

$$
\left\|y_{n}-g_{n}\right\| \leqslant C_{2}(n+1)^{-1}
$$

для всех $n=0,1,2, \ldots$.

ДокАЗАТЕЛЬСтво. Положим $D=\max _{s \in[0, p]}\left|f\left(s^{2}\right)\right|$ и докажем, что

$$
\left|\lambda_{n}-z_{n}\right| \leqslant D
$$

для всех $n \geqslant 0$. Предположим, что $\lambda_{n}>z_{n}+D$ для некоторого $n$, и сравним задачу (1)-(2) при $\lambda=\lambda_{n}$ с задачей

$$
-e_{n}^{\prime \prime}=z_{n} e_{n}, \quad e_{n}^{\prime}(0)=e_{n}(A)=0
$$

Тогда в силу утверждения (в) теоремы 1 и (6) $\lambda_{n}-f\left(y_{n}^{2}(x)\right) y_{n}(x)>z_{n}$ для этого номера $n$ и всех $x \in(0, A)$. Но тогда в силу известной теоремы сравнения (см., например, [9]) функция $y_{n}(x)$ имеет в интервале $(0, A)$ не менее $n+1$ корней (согласно стандартным результатам между каждыми двумя корнями функции $e_{n}(x)$ лежит корень функции $y_{n}(x)$ и в интервале $(0, A /(2 n+1))$, где $A /(2 n+1)$ - минимальный положительный корень функции $e_{n}$, лежит по крайней мере один корень функции $y_{n}$ ), что противоречиво. Поэтому $\lambda_{n} \leqslant z_{n}+D$ для всех $n$. Аналогично можно доказать, что $\lambda_{n} \geqslant z_{n}-D$. Тем самьм (10) доказано.

В силу (6) и (10) получаем, что функции $u_{n}(x)=y_{n}(x)-g_{n}(x)$ удовлетворяют задачам

$$
\begin{gathered}
-u_{n}^{\prime \prime}(x)+U_{n}(x)=z_{n} u_{n}(x), \quad x \in(0, A), \\
u_{n}(0)=u_{n}^{\prime}(0)=u_{n}(A)=0
\end{gathered}
$$

где непрерывные функции $U_{n}(x)=-\left(\lambda_{n}-z_{n}\right) y_{n}(x)+f\left(y_{n}^{2}(x)\right) y_{n}(x)(n=0,1,2, \ldots)$ образуют последовательность равномерно ограниченную на отрезке $x \in[0, A]$, поскольку ввиду теоремы 1(в) и оценки (6) имеем $\left|y_{n}(x)\right| \leqslant p$ для всех $n$ и $x$.

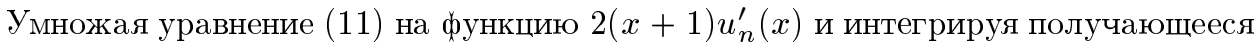
тождество по отрезку $[0, A]$ с применением формулы интегрирования по частям, в силу (12) и неравенства $2 a b \leqslant a^{2}+b^{2}$ получаем:

$$
\begin{aligned}
z_{n} \int_{0}^{A} u_{n}^{2}(x) d x & =-2 \int_{0}^{A}(x+1) u_{n}^{\prime}(x) U_{n}(x) d x-\int_{0}^{A}\left[u_{n}^{\prime}(x)\right]^{2} d x+(A+1)\left[u_{n}^{\prime}(A)\right]^{2} \\
& \leqslant \int_{0}^{A}(x+1)^{2} U_{n}^{2}(x) d x+(A+1)\left[u_{n}^{\prime}(A)\right]^{2} \\
& \leqslant C_{3}+(A+1)\left[u_{n}^{\prime}(A)\right]^{2}
\end{aligned}
$$

где $C_{3}>0$ не зависит от номера $n=0,1,2, \ldots$ 
Далее, поскольку для любого целого $n \geqslant 0$ функции $y_{n}(x)$ и $g_{n}(x)$ имеют одинаковое число корней в интервале $(0, A)$, знаки величин $y_{n}^{\prime}(A)$ и $g_{n}^{\prime}(A)$ совпадают. Кроме того, $\left[g_{n}^{\prime}(A)\right]^{2}=z_{n} p^{2}$. Используя эти свойства, а также оценку (10) и тождество (5), получаем, что существует $C_{4}>0$ такое, что

$$
\left|u_{n}^{\prime}(A)\right| \leqslant C_{4}
$$

для всех номеров $n=0,1,2, \ldots$ Из (13) и (14) следует, что существует $C_{5}>0$ такое, что

$$
z_{n} \int_{0}^{A} u_{n}^{2}(x) d x \leqslant C_{5}
$$

для всех номеров $n$. Тем самым лемма 4 доказана.

Справедливость условия Б теоремы Бари в пространстве $L_{2}$ для систем функций $\left\{e_{n}\right\}_{n=0}^{\infty}$ и $\left\{w_{n}\right\}_{n=0}^{\infty}$ вытекает из доказанной леммы 4. Поэтому система функций $\left\{w_{n}\right\}_{n=0}^{\infty}$ является базисом Бари, а система функций $\left\{y_{n}\right\}_{n=0}^{\infty}-$ базисом Рисса в пространстве $L_{2}$, и теорема 1 полностью доказана.

\section{Список литературы}

1. Камке Э. Справочник по обыкновенным дифференциальньтм уравнениям. М.: Наука, 1976.

2. Жидков П. Е. О полноте систем собственных функций оператора Штурма-Лиувилля с потенциалом, зависящим от спектрального параметра, и некоторой нелинейной задачи // Матем. сб. 1997. Т. 188. № 7. С. 123-138.

3. Zhidkov P. E. Eigenfunction expansions associated with a nonlinear Schrödinger equation // Joint Inst. Nuclear Research Comm. E5-98-61. Dubna. 1998.

4. Бари Н. К. О базисах в гильбертовом пространстве // Докл. АН СССР. 1946. Т. 54. № 5. C. $383-386$.

5. Бари H. К. Биортогоналњные системы и базисы в гильбертовом пространстве // Ученые записки МГУ. Математика. 1951. Т. 148. №4. С. 69-107.

6. Гутер Р. С., Ульянов П. Л. О новых результатах в теории ортогональных рядов // Дополнение в книге Качмаж С., Штейнгауз Г. Теория ортогональных рядов. М.: Гос. изд. физ.-мат. лит-ры, 1958.

7. Гохберг И. Ц., Крейн М. Г. Введение в теорию линейных несамосопряженных операторов. М.: Наука, 1965.

8. Махмудов А. П. Основы нелинейного спектрального анализа. Баку: Изд-во Азербайджанского гос. ун-та им. С.М. Кирова, 1984.

9. Левитан Б. М., Саргсян И. С. Операторы Штурма-Лиувилля и Дирака. М.: Наука, 1988.

10. Люстерник Л.А., Соболев В.И. Элементы функционалњного анализа. М.: Наука, 1965 .

Объединенньй институт ядерных исследований, г. Дубна

Поступила в редакцию

E-mail: zhidkov@thsun1.jinr.ru

03.11.1998 и 14.07 .1999 